En réalité, c'en est fascinant: on trouve au sein du corps médical une variété d'opinions politiques qu'on ne constate sans doute dans aucun autre groupe professionnel.

Reste à savoir si les années qui passent atténuent certains clivages? Les textes qui paraissent dans le présent numéro du BMS et qui sont consacrés à la lutte contre les armes nucléaires auraient certainement provoqué une vive polémique il y a quelques décennies - le point de vue qu'ils défendent est aujourd'hui pour beaucoup une évidence, et je doute qu'il soulève encore beaucoup de vagues. Mais il n'empêche! Pour une profession qui lutte pour la vie, et pour une vie qui puisse garder de bonnes conditions aussi longtemps que possible, l'idée que face aux armes nucléaires il n'y a pas de soins ni même de médecine palliative possibles, comme le disent les auteurs de l'article ci-dessous, et que seule la prévention a un sens, est une idée qui devrait être motivante et pousser à la réflexion, ... à l'action.

Je souhaite beaucoup de succès au Congrès mondial des Médecins pour la prévention de la guerre nucléaire; je souhaite surtout qu'au-delà de la confortable indifférence de beaucoup, il soit l'occasion de réveiller quelques consciences!

Jacques de Haller, Président de la FMH

\title{
Pourquoi le corps médical suisse doit aussi s'intéresser à la bombe atomique et à la radioactivité
}

\author{
Günter Baitsch ${ }^{a}$, \\ Claudio Knüsli ${ }^{b}$, \\ Jacques Moserc, \\ Andreas Nideckerd, \\ Martin Waltere
a Médecine interne, Bâle
b Oncologie, Bâle
c Médecine interne, Lausanne
d Radiologie, Bâle \\ e Médecine interne, Granges
}

\footnotetext{
* International Physicians for the Prevention of Nuclear War / Médecins internationaux pour la prévention de la guerre nucléaire
}

(Version française: Jacques Moser)

Correspondance: Prof. Andreas Nidecker c/o Imamed Radiologie Nordwest Untere Rebgasse 18 CH-4058 Bâle

andreas.nidecker@imamed.ch
"Le centre de la ville était une sorte de tache blanche, polie comme la paume de la main (...) des milliers d'être humains dans les rues et les jardins, frappés par une vague de chaleur aiguë, meurent comme des mouches sous l'effet de la température. D'autres se tordent comme des vers, atrocement brûlés...»

Voici ce qu'on peut lire dans le rapport du docteur Marcel Junod, premier délégué du CICR arrivé à Hiroshima en 1945.

\section{De la folie à la «normalité»}

L'opinion publique en est peu consciente, mais le risque de guerre nucléaire est aussi élevé que jamais; la situation actuelle est même, selon certains experts, particulièrement critique. Il y a plusieurs raisons à cela, à commencer par le nombre d'armes sur la planète. En effet, malgré les efforts de désarmement entrepris dès les années 80 , l'arsenal est encore de 23000 armes atomiques balistiques. Parmi elles, un grand nombre est en état d'alerte dit «hair-trigger-alert», - principalement en Russie et aux Etats-Unis - et peut être largué en quelques minutes. Ces missiles sont des symboles de puissance. Les chefs d'Etat aiment se pavaner sur des sous-marins nucléaires, même si ces armes n'ont pas apporté beaucoup de prestige au Pakistan et à la Corée du Nord.

Certaines grandes puissances européennes, sûres d'elles, affirment ne pouvoir garantir la sécurité de leur population que par l'armement atomique, alors que d'autres, dont les besoins sont les mêmes, n'ont jamais choisi cette option. Il ne faut pas s'étonner que de petits Etats aspirent aussi à la bombe: les mauvais exemples font des émules. L'évolution est inquiétante sur la presqu'île coréenne, dans le sous-continent indien et au Moyen-Orient. La Corée du Nord, un Etat nucléaire, a explicitement menacé de recourir à la bombe. Le Pakistan, politiquement instable, avec des zones tenues par la rébellion, en conflit permanent avec l'Inde au sujet du Cachemire (ce qui, en 1999, a failli dégénérer en escalade nucléaire), dispose d'un appareil militaire ayant la main sur le nucléaire. L'Iran est un pays instable, potentiellement un Etat nucléaire. Israël, un Etat nucléaire non déclaré, détient vraisemblablement des plans de destruction des installations atomiques iraniennes. Les puissances nucléaires traditionnelles, - France, Etats-Unis, Grande Bretagne, Russie, Chine - renouvellent leurs arsenaux. La réduction du nombre des têtes nucléaires n'empêche pas la modernisation. Au mieux, on réduit légèrement l'énorme surcapacité à détruire toute vie sur terre («Overkill»). Quant au Traité de non-prolifération des armes atomiques, il est actuellement en révision. Les résultats ne sont pas encore connus.

Le risque de guerre atomique est également augmenté par l'informatisation du secteur militaire: chacun sait que même les meilleurs ordinateurs peuvent dysfonctionner. Quant au complexe militaro-industriel, il a tout intérêt à maintenir le statu quo et, pour s'assurer des profits, il ne manque pas d'arguments. Toutes ces évolutions ne sont guère perçues par la société qui les considère comme normales. 


\section{La prévention, un devoir}

Nous ne pouvons pas aider les victimes d'une attaque nucléaire: il n'y a même plus de médecine palliative possible, parce que toutes les infrastructures sont détruites. Les survivants d'une attaque nucléaire meurent lentement et sans bruit. La seule attitude responsable pour un médecin humaniste et socialement engagé est de prévenir, c'est-à-dire d'œuvrer en faveur de l'abolition de toutes les armes atomiques. Ce n'est autre que de $l^{\prime}$ «Evidence Based Medicine». Certes, un monde sans armes atomiques ne sera pas réalisé du jour au lendemain, mais les efforts diplomatiques doivent être poursuivis, sans oublier le rôle important que peuvent jouer les ONG et la société civile.

\section{Le faible rayonnement, une autre menace pour la santé}

Le rayonnement de faible intensité affecte également la santé. Les populations d'une bonne dizaine de pays qui exploitent des mines d'uranium sont exposées à des risques permanents: les nappes phréatiques sont polluées, les vents emportent les sables contaminés, et l'accès à l'uranium engendre des guerres. Les problèmes de santé des personnes qui ont été exposées à une explosion atomique, que ce soit à Hiroshima, à Nagasaki ou lors de tests dans le Nevada, au Kazakhstan, en Chine, en Polynésie ou en Algérie (où des soldats français ont été inclus à leur insu dans une étude longitudinale), mettent en évidence qu'aucune prévention secondaire n'est possible. Il faudra également

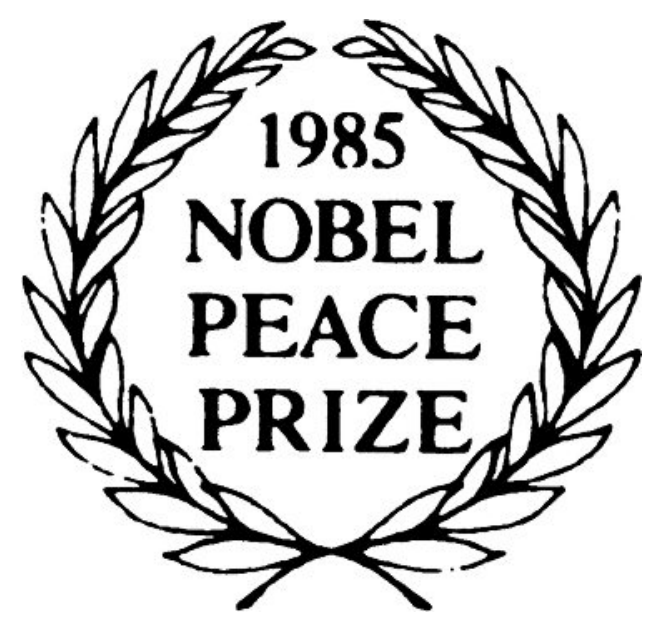

répondre, avec la plus grande rigueur scientifique, à la question de savoir si le faible rayonnement à proximité des centrales nucléaires augmente le risque de leucémie infantile.

La section suisse des Médecins Internationaux pour la Prévention de la Guerre nucléaire (International Physicians for the Prevention of Nuclear War IPPNW) organise à Bâle cet été le 19 ème Congrès mondial IPPNW. L'événement sera également l'occasion de commémorer le $25^{\text {ème }}$ anniversaire de la remise du Prix Nobel de la Paix à IPPNW en 1985.

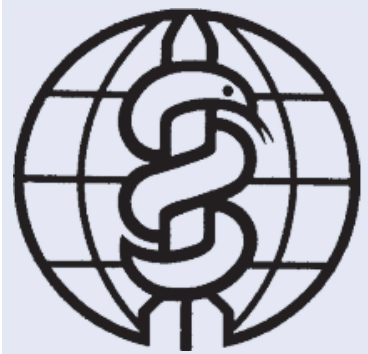

19e Congrès mondial IPPNW à Bâle

Tou(te)s les collègues sont cordialement invités à assister au Congrès mondial IPPNW qui se tiendra du 25 au 29 août 2010 à I'Université de Bâle. Les thèmes mentionnés dans l'article ci-contre, et d'autres sujets apparentés, seront abordés par des personnalités compétentes du monde entier. Un papillon annonçant le congrès est annexé au présent numéro du Bulletin des médecins suisses. Vous pouvez également consulter notre site internet www.ippnw2010.org 